\title{
THE CORED AND LOGARITHM GALACTIC POTENTIALS: PERIODIC ORBITS AND INTEGRABILITY
}

\author{
LIDIA JIMÉNEZ-LARA ${ }^{1}$ AND JAUME LLIBRE ${ }^{2}$
}

Abstract. We apply the averaging theory of first order to study analytically families of periodic orbits for the cored and logarithmic Hamiltonians

$$
H_{C}=\frac{1}{2}\left(p_{x}^{2}+p_{y}^{2}\right)+\sqrt{1+x^{2}+\frac{y^{2}}{q^{2}}}-1,
$$

and

$$
H_{L}=\frac{1}{2}\left(p_{x}^{2}+p_{y}^{2}\right)+\frac{1}{2} \log \left(1+x^{2}+\frac{y^{2}}{q^{2}}\right),
$$

which are relevant in the study of the galactic dynamic. We first show, after introducing a scale transformation in the coordinates and momenta with a parameter $\varepsilon$, that both systems give essentially the same set of equations of motion up to first order in $\varepsilon$. Then the conditions for finding families of periodic orbits, using the averaging theory up to firs order in $\varepsilon$, apply equally for both systems in every energy level $H=h>0$ with $H$ either $H_{C}$ or $H_{L}$. We prove the existence of two periodic orbits if $q$ is irrational, for $\varepsilon$ small enough, and we give an analytic approximation for the initial conditions of these periodic orbits. Finally, the previous periodic orbits provide information about the non-integrability of the cored and the logarithmic Hamiltonian systems.

\section{INTRODUCTION AND STATEMENT OF THE MAIN RESUltS}

We study the following two potentials

(1)

$$
\begin{aligned}
& V_{C}=\sqrt{R^{2}+x^{2}+y^{2} / q^{2}}-R \\
& V_{L}=\frac{1}{2} \log \left(R^{2}+x^{2}+y^{2} / q^{2}\right)
\end{aligned}
$$

Date: February 29, 2012.

Key words and phrases. periodic orbits, integrability, logarithmic potential, cored potential, averaging theory. 
called the cored and logarithmic potentials, respectively, which have an absolute minimum and reflection symmetry with respect to both axes. These potentials are of interest in problems of galactic dynamics as models for elliptical galaxies.

The logarithmic potential is a model of a core embedded in a dark matter halo, with $R$ the core radius [11]. Papaphilippou and Laskar [10] use the Laskar's frequency map analysis applied to study the numerically integrated orbits, giving a global vision of the dynamics in the phase space. Contopoulos and Seimenis [6] have applied the Prendergast method to approximate solutions of the logarithmic potential in the form of rational functions. An analogous method to the Pendergrast based in series expansions computed by inverting the normalizing canonical transformation was used by Pucacco et. al. [12] to find periodic orbits. The structure of the phase space related to the logarithmic potential has been approximated with resonant detuned normal forms constructed with the method based on the Lie transform by Pucacco et.al. [11, 12] and Belmonte et. al. [4]. Detuned resonant normal forms allow to study several features of the non-linear oscillations. The existence of periodic orbits associated to the non-linear resonant normal modes is assumed on the basis of the symmetry of the potential. Here we give a formal proof of their existence.

We can choose $R=1$ without loss of generality, and the energy may take in both cases any non-negative value. The parameter $q$ gives the ellipticity of the potential, which ranges in the interval $0.6 \leq q \leq 1$. Lower values of $q$ have no physical meaning, and greater values of $q$ are equivalent to reverse the role of the coordinate axes.

It is well known that integrable and non-integrable Hamiltonian systems can have infinitely many periodic orbits. However it is difficult to find a whole family of periodic orbits in an analytical way, specially if the Hamiltonian system is non-integrable. Here we find two families in every positive level of the Hamiltonian.

We start with the Hamiltonian systems associated to the cored and logarithmic potentials (1), both having the first integral given by the total energy $H_{C}=K+V_{C}$ and $H_{L}=K+V_{L}$, respectively

$$
H_{C}=\frac{1}{2}\left(p_{x}^{2}+p_{y}^{2}\right)+\sqrt{1+x^{2}+\frac{y^{2}}{q^{2}}}-1,
$$


whose cored Hamiltonian system is

$$
\begin{aligned}
\dot{x} & =\frac{\partial H_{C}}{\partial p_{x}}=p_{x}, \\
\dot{y} & =\frac{\partial H_{C}}{\partial y}=p_{y}, \\
\dot{p}_{x} & =-\frac{\partial H_{C}}{\partial x}=-\frac{x}{\sqrt{1+x^{2}+\frac{y^{2}}{q^{2}}}} \\
\dot{p}_{y} & =-\frac{\partial H_{C}}{\partial y}=-\frac{y}{q^{2} \sqrt{1+x^{2}+\frac{y^{2}}{q^{2}}}} .
\end{aligned}
$$

The logarithmic Hamiltonian is

$$
H_{L}=\frac{1}{2}\left(p_{x}^{2}+p_{y}^{2}\right)+\frac{1}{2} \log \left(1+x^{2}+\frac{y^{2}}{q^{2}}\right),
$$

whose logarithmic Hamiltonian system is given by

$$
\begin{aligned}
\dot{x} & =\frac{\partial H_{L}}{\partial p_{x}}=p_{x}, \\
\dot{y} & =\frac{\partial H_{L}}{\partial y}=p_{y} \\
\dot{p}_{x} & =-\frac{\partial H_{L}}{\partial x}=-\frac{x}{1+x^{2}+\frac{y^{2}}{q^{2}}}, \\
\dot{p}_{y} & =-\frac{\partial H_{L}}{\partial y}=-\frac{y}{q^{2}\left(1+x^{2}+\frac{y^{2}}{q^{2}}\right)} .
\end{aligned}
$$

We first show in section 3 that, after introducing a non-canonical scale transformation in the coordinates and momenta with a parameter $\varepsilon$ of the form

$$
\left\{x, y, p_{x}, p_{y}\right\} \rightarrow\left\{\sqrt{\varepsilon} x, \sqrt{\varepsilon} y, \sqrt{\varepsilon} p_{x}, \sqrt{\varepsilon} p_{y}\right\}
$$


both Hamiltonian systems (3) and (5) can be reduced to study the differential system

$$
\begin{aligned}
\dot{x} & =p_{x} \\
\dot{y} & =p_{y} \\
\dot{p}_{x} & =-x+\frac{\varepsilon x}{2}\left(x^{2}+\frac{y^{2}}{q^{2}}\right)+\mathcal{O}\left(\varepsilon^{2}\right), \\
\dot{p}_{y} & =-\frac{y}{q^{2}}+\frac{\varepsilon y}{2 q^{4}}\left(q^{2} x^{2}+y^{2}\right)+\mathcal{O}\left(\varepsilon^{2}\right),
\end{aligned}
$$

having the first integral

$$
H=\frac{1}{2}\left(p_{x}^{2}+p_{y}^{2}+x^{2}+\frac{y^{2}}{q^{2}}\right)-\varepsilon \frac{\left(q^{2} x^{2}+y^{2}\right)^{2}}{8 q^{4}}+\mathcal{O}\left(\varepsilon^{2}\right) .
$$

Thus, the conditions for finding families of periodic orbits using the averaging theory up to first order in $\varepsilon$, apply equally for both systems.

We apply the averaging theory of first order in the small parameter $\varepsilon$ to compute periodic orbits of a perturbed periodic differential system depending on $\varepsilon$. We recall in section 2 the basic theorem of this tool: the Averaging Theorem of first order. This theorem provides, under certain conditions, perturbed periodic orbits for $\varepsilon$ sufficiently small that bifurcate from some unperturbed periodic orbits for $\varepsilon=0$. The method goes back to Malkin [13] and Roseau [15], and a shorter proof is given by Buică et. al. [5]. For a general introduction to the averaging theory see the books of Sanders, Verhulst and Murdock [16], and of Verhulst [18].

We find two families of periodic orbits parametrized by the energy when the parameter $q$ is irrational, all of them bifurcating from unperturbed periodic orbits around the center: one bifurcating from the two dimensional plane $\left(x, 0, p_{x}, 0\right)$, and another one from the two dimensional plane $\left(0, y, 0, p_{y}\right)$. When $q$ is rational, the Averaging Theorem gives no information about periodic orbits.

Our main results on the periodic orbits of the cored and logarithmic Hamiltonian systems are summarized in the next two theorems, which are proved in sections 4 and 5 , respectively.

Theorem 1. For $\varepsilon>0$ sufficiently small and $q$ irrational, at every energy level $H=h>0$ the perturbed differential system (6) has at least one periodic solution $\left(x(t), y(t), p_{x}(t), p_{y}(t)\right)$ such that $\left(x(0), y(0), p_{x}(0), p_{y}(0)\right) \rightarrow$ $(\sqrt{2 h}, 0,0,0)$. 
Theorem 2. For $\varepsilon>0$ sufficiently small and $q$ irrational, at every energy level $H=h>0$ the perturbed differential system (6) has at least one periodic solution $\left(x(t), y(t), p_{x}(t), p_{y}(t)\right)$ such that $\left(x(0), y(0), p_{x}(0), p_{y}(0)\right) \rightarrow$ $(0, \sqrt{2 h} q, 0,0)$.

Putting together Theorems 1 and 2 we shall have at every energy level $H=h>0$ at least two periodic orbits if $q$ is irrational.

The case $q$ rational was also studied in cartesian coordinates but we did not obtain information because we could not solve the variational equations. Working with a generalization of polar coordinates, we tried to find isolated periodic solutions, but the averaging method did not give information.

Finally, the existence of these two families of periodic orbits found with averaging theory give sufficient conditions in order to claim that there cannot exist any second first integral of class $\mathcal{C}^{1}$ whose gradient be independent with the Hamiltonian gradient on the points of some of the periodic orbits that we have found.

Theorem 3. For the cored and logarithmic Hamiltonian systems (3) and (5) with $q$ irrational,

(a) either they are not Liouville-Arnold integrable with any second first integral $\mathcal{C}^{1}$,

(b) or they are Liouville-Arnold integrable with a second first integral $C$ and the gradients of $H$ and $C$ are linearly dependent on some point for every periodic orbit found in Theorems 1 and 2.

As the case (b) of Theorem 3 is exceptional, the case (a) holds generically, see for more details section 6 . This is important because most of the results about integrability deals with analytic or meromorphic integrals of motion.

Theorem 3 is proved in section 6 , where the definition of integrability in the sense of Liouville-Arnold is also recalled. Similar results to Theorems 1 and 2 were obtained by the authors for the Henon-Heiles [9] and YangMills [8] Hamiltonian systems with 2 parameters using the averaging theory of second and first order, respectively. Besides, as it is established in [9], the averaging method for finding isolated periodic orbits is an useful and relatively simple tool in order to find necessary conditions for showing the non-integrability of a Hamiltonian system. 
We use a result due to Poincaré $[2,7,14]$ for proving the non LiouvilleArnold integrability, based on the existence of the periodic orbits of Theorems 1 and 2 having multipliers of the monodromy matrix of the variational equations different from 1.

\section{The AVERAging THEORY OF FIRST ORDER}

In this section we recall the basic results from averaging theory of first order that we shall need for proving the main results of this paper.

We consider the problem of the bifurcation of T-periodic solutions from the differential system

$$
\dot{\mathbf{x}}(t)=F_{0}(t, \mathbf{x})+\varepsilon F_{1}(t, \mathbf{x})+\varepsilon^{2} F_{2}(t, \mathbf{x}, \varepsilon),
$$

with $\varepsilon \neq 0$ sufficiently small. Here the functions $F_{0}, F_{1}: \mathbb{R} \times \Omega \rightarrow \mathbb{R}^{n}$ and $F_{2}: \mathbb{R} \times \Omega \times\left(-\varepsilon_{f}, \varepsilon_{f}\right) \rightarrow \mathbb{R}^{n}$ are $\mathcal{C}^{2}$ functions, T-periodic in the first variable, and $\Omega$ is an open subset of $\mathbb{R}^{n}$. One of the main assumptions is that the unperturbed system

$$
\dot{\mathbf{x}}(t)=F_{0}(t, \mathbf{x})
$$

has a $k$-dimensional submanifold of $T$-periodic solutions. We assume that the coordinates have been taken in such a way that the $k$-dimensional submanifold of periodic orbits is contained in $\left\{\left(x_{1}, \ldots, x_{k}, 0, \ldots, 0\right) \in\right.$ $\Omega\}$. A solution of this problem is given using the averaging theory.

Let $\mathbf{x}(t, \mathbf{z})$ be the solution of the unperturbed system (9) such that $\mathbf{x}(0, \mathbf{z})=\mathbf{z}$. We write the linearization of the unperturbed system along the periodic solution $\mathbf{x}(t, \mathbf{z})$ as

$$
\dot{\mathbf{y}}(t)=D_{\mathbf{x}} F_{0}(t, \mathbf{x}(t, \mathbf{z})) \mathbf{y} .
$$

In what follows we denote by $M_{\mathbf{z}}(t)$ some fundamental matrix of the linear differential system (10), and by $\xi: \mathbb{R}^{k} \times \mathbb{R}^{n-k} \rightarrow \mathbb{R}^{k}$ the projection of $\mathbb{R}^{n}$ onto its first $k$ coordinates; i.e. $\xi:\left(x_{1}, \ldots, x_{n}\right)=\left(x_{1}, \ldots, x_{k}\right)$.

Averaging Theorem (first order). Let $V \subset \mathbb{R}^{k}$ be open and bounded, and let $\beta_{0}: \mathrm{Cl}(V) \rightarrow \mathbb{R}^{n-k}$ be a $\mathcal{C}^{2}$ function. We assume that

(i) $\mathcal{Z}=\left\{\mathbf{z}_{\alpha}=\left(\alpha, \beta_{0}(\alpha)\right), \alpha \in \mathrm{Cl}(V)\right\} \subset \Omega$ and that for each $\mathbf{z}_{\alpha} \in \mathcal{Z}$ the solution $\mathbf{x}\left(t, \mathbf{z}_{\alpha}\right)$ of (9) is T-periodic;

(ii) for each $\mathbf{z}_{\alpha} \in \mathcal{Z}$ there is a fundamental matrix $M_{\mathbf{z}_{\alpha}}(t)$ of (10) such that the matrix $M_{\mathbf{z}_{\alpha}}^{-1}(0)-M_{\mathbf{z}_{\alpha}}^{-1}(T)$ has in the upper right corner the $k \times(n-k)$ zero matrix, and in the lower right corner $a(n-k) \times(n-k)$ matrix $\Delta_{\alpha}$ with $\operatorname{det} \Delta_{\alpha} \neq 0$. 
We consider the function $\mathcal{F}: \mathrm{Cl}(V) \rightarrow \mathbb{R}^{k}$

$$
\mathcal{F}(\alpha)=\xi\left(\int_{0}^{T} M_{\mathbf{z}_{\alpha}}^{-1}(t) F_{1}\left(t, \mathbf{x}\left(t, \mathbf{z}_{\alpha}\right)\right) d t\right) .
$$

If there exists $a \in V$ with $\mathcal{F}(a)=0$ and

$$
\operatorname{det}((d \mathcal{F} / d \alpha)(a)) \neq 0,
$$

then there is a T-periodic solution $\varphi(t, \varepsilon)$ of system (8) such that $\varphi(0, \varepsilon) \rightarrow$ $\mathbf{z}_{\alpha}$ as $\varepsilon \rightarrow 0$.

We briefly repeat the proof given in [5]. The idea is to find the zeros $\mathbf{z}_{\alpha}$ of the function $f(\mathbf{z}, \varepsilon)=\mathbf{x}(T, \mathbf{z}, \varepsilon)-\mathbf{z}$ or equivalently of $g(\mathbf{z}, \varepsilon)=$ $M_{\mathbf{z}}^{-1}(T) f(\mathbf{z}, \varepsilon)$, since they provide periodic orbits of the perturbed system. The authors first show that

$$
\frac{d g}{d \mathbf{z}}\left(\mathbf{z}_{\alpha}, 0\right)=M_{\mathbf{z}_{\alpha}}^{-1}(0)-M_{\mathbf{z}_{\alpha}}^{-1}(T) .
$$

From the definition of $g(\mathbf{z}, \varepsilon)$

$$
\frac{\partial g}{\partial \varepsilon}(\mathbf{z}, 0)=M_{\mathbf{z}}^{-1}(T) \frac{\partial \mathbf{x}}{\partial \varepsilon}(T, \mathbf{z}, 0) .
$$

The function $(\partial \mathbf{x} / \partial \varepsilon)(t, \mathbf{z}, 0)$ is the unique solution of the initial value problem

$$
\mathbf{y}^{\prime}=D_{\mathbf{x}} F_{0}(t, \mathbf{x}(t, \mathbf{z}, 0)) \mathbf{y}+F_{1}(t, \mathbf{x}(t, \mathbf{z}, 0)), \quad \mathbf{y}(0)=0,
$$

then

$$
\frac{\partial \mathbf{x}}{\partial \varepsilon}(t, \mathbf{z}, 0)=M_{\mathbf{z}}(t) \int_{0}^{t} M_{\mathbf{z}}^{-1}(s) F_{1}(s, \mathbf{x}(t, \mathbf{z}, 0)) d s .
$$

The equation (13) results in

$$
\frac{\partial g}{\partial \varepsilon}(\mathbf{z}, 0)=\int_{0}^{T} M_{\mathbf{z}}^{-1}(s) F_{1}(s, \mathbf{x}(t, \mathbf{z}, 0)) d s .
$$

Then

$$
\frac{\partial(\xi g)}{\partial \varepsilon}\left(\mathbf{z}_{\alpha}, 0\right)=\xi\left(\int_{0}^{T} M_{\mathbf{z}_{\alpha}}^{-1}(t) F_{1}\left(t, \mathbf{x}\left(t, \mathbf{z}_{\alpha}\right)\right) d t\right) .
$$

Finally, the authors show that there exist $\alpha_{\varepsilon} \in V$ such that $g\left(\mathbf{z}_{\alpha_{\varepsilon}}, \varepsilon\right)=0$ and $f\left(\mathbf{z}_{\alpha_{\varepsilon}}, \varepsilon\right)=0$, then the solution $\mathbf{x}\left(t, \mathbf{z}_{\alpha_{\varepsilon}}, \varepsilon\right)$ is periodic.

The case when $k=n$ is called perturbations of an isochronous set and it was proved in Corollary 1 of Buică et. al. of [5]. We tried to use this corollary to study the case $q$ rational, but we could not get positive information in this case. 


\section{The scale transformation and the First order DIFFERENTIAL SYSTEM}

Of course, the Hamiltonian systems (3) and (5) are not into the normal form (8) for applying the averaging theory. So we first introduce a noncanonical rescaling transformation with a factor $\sqrt{\varepsilon}$ in order to have a small parameter $\varepsilon>0$ in the differential system

$$
\left\{x, y, p_{x}, p_{y}\right\} \rightarrow\left\{\sqrt{\varepsilon} x, \sqrt{\varepsilon} y, \sqrt{\varepsilon} p_{x}, \sqrt{\varepsilon} p_{y}\right\}
$$

We will show that both the cored and logarithmic systems provide the same differential system up to first order in $\varepsilon$, then the conditions for finding families of periodic orbits using the averaging theory shall apply equally for both systems.

We first consider the differential system (3) of the cored potential in the rescaled variables

$$
\begin{aligned}
\dot{x} & =p_{x}, \\
\dot{y} & =p_{y}, \\
\dot{p}_{x} & =-\frac{x}{\sqrt{1+\varepsilon\left(x^{2}+\frac{y^{2}}{q^{2}}\right)}}, \\
\dot{p}_{y} & =-\frac{y}{q^{2} \sqrt{1+\varepsilon\left(x^{2}+\frac{y^{2}}{q^{2}}\right)}},
\end{aligned}
$$

with the first integral

$$
H=\frac{\varepsilon}{2}\left(p_{x}^{2}+p_{y}^{2}\right)+\sqrt{1+\varepsilon\left(x^{2}+\frac{y^{2}}{q^{2}}\right)} .
$$

As the change to the new variables is only a rescaling transformation, the differential system (14) for all $\varepsilon>0$ is topologically equivalent to the cored Hamiltonin system (3). Therefore studying the differential system (14) for small values of $\varepsilon \neq 0$, we are also studying the original cored Hamiltonian system (3) with $\varepsilon=1$.

The differential system (14) has a unique singular point, the origin. 
Now we expand equations (14) in powers of the small parameter $\varepsilon$ and the first integral $H$ up to first order in $\varepsilon$, thus we have,

$$
\begin{aligned}
\dot{x} & =p_{x}, \\
\dot{y} & =p_{y}, \\
\dot{p}_{x} & =-x+\frac{\varepsilon x}{2}\left(x^{2}+\frac{y^{2}}{q^{2}}\right)+\mathcal{O}\left(\varepsilon^{2}\right), \\
\dot{p}_{y} & =-\frac{y}{q^{2}}+\frac{\varepsilon y}{2 q^{4}}\left(q^{2} x^{2}+y^{2}\right)+\mathcal{O}\left(\varepsilon^{2}\right),
\end{aligned}
$$

and the first integral becomes

$$
K=1+\frac{\varepsilon}{2}\left(p_{x}^{2}+p_{y}^{2}+x^{2}+\frac{y^{2}}{q^{2}}\right)-\varepsilon^{2} \frac{\left(q^{2} x^{2}+y^{2}\right)^{2}}{8 q^{4}}+\mathcal{O}\left(\varepsilon^{3}\right) .
$$

As $K$ is a first integral, also $H=(K-1) / \varepsilon$ is a first integral, and we are going to use this first integral

$$
H=\frac{1}{2}\left(p_{x}^{2}+p_{y}^{2}+x^{2}+\frac{y^{2}}{q^{2}}\right)-\varepsilon \frac{\left(q^{2} x^{2}+y^{2}\right)^{2}}{8 q^{4}}+\mathcal{O}\left(\varepsilon^{2}\right) .
$$

If we do the same with the logarithmic Hamiltonian system (5) in the rescaled variables, we obtain the same differential equations (16) but with $\varepsilon / 2$ instead of $\varepsilon$. Then we shall consider only the differential system (16), and our results will apply equally for the cored and logarithmic Hamiltonian systems.

The unperturbed equations with $\varepsilon=0$ represent a bidimensional harmonic oscillator that can be easily solved with arbitrary initial conditions $x(0)=x_{0}, y(0)=y_{0}, p_{x}(0)=p_{x_{0}}, p_{y}(0)=p_{y_{0}}$

$$
\begin{aligned}
x(t) & =x_{0} \cos t+p_{x_{0}} \sin t, \\
p_{x}(t) & =p_{x_{0}} \cos t-x_{0} \sin t, \\
y(t) & =y_{0} \cos \left(\frac{t}{q}\right)+p_{y_{0}} q \sin \left(\frac{t}{q}\right), \\
p_{y}(t) & =p_{y_{0}} \cos \left(\frac{t}{q}\right)-\frac{y_{0}}{q} \cos \left(\frac{t}{q}\right) .
\end{aligned}
$$

If $q$ is irrational, the periodic orbits of the unperturbed system are contained in two planes: one at the two dimensional plane $\left(x, 0, p_{x}, 0\right)$, and another at the two dimensional plane $\left(0, y, 0, p_{y}\right)$.

If $q$ is rational, the unperturbed periodic orbits live in the four dimensional phase space. However, we could not find periodic orbits with the averaging method of section 2 , and only the irrational case of $q$ will be studied in this paper. 


\section{Proof of Theorem 1}

We consider the case $q$ irrational and the unperturbed periodic orbit at the plane $\left(x, 0, p_{x}, 0\right)$

$$
\begin{aligned}
x(t) & =x_{0} \cos t+p_{x_{0}} \sin t, \\
p_{x}(t) & =p_{x_{0}} \cos t-x_{0} \sin t, \\
y(t) & =0, \\
p_{y}(t) & =0,
\end{aligned}
$$

with the first integral (17) when $\varepsilon=0$ taking the energy value

$$
h=\left(p_{x_{0}}^{2}+x_{0}^{2}\right) / 2 .
$$

Generically, the periodic orbits of a Hamiltonian system with more than one degree of freedom are on cylinders filled of periodic orbits. Therefore we cannot apply directly the Averaging Theorem to the Hamiltonian system, since the determinant (12) would be always zero. Then we must apply Averaging Theorem to every Hamiltonian fixed level where the periodic orbits generically are isolated. This allows to eliminate one of the coordinates, say $p_{x}$, and to reduce the study to dimension 3 .

We thus compute $p_{x}$ at the energy level $H=h$ with $h$ given by (17)

$$
p_{x}= \pm \sqrt{x_{0}^{2}+p_{x_{0}}^{2}-x^{2}-p_{y}^{2}-\frac{y^{2}}{q^{2}}+\varepsilon\left(\frac{x^{4}}{4}+\frac{x^{2} y^{2}}{2 q^{2}}+\frac{y^{4}}{4 q^{4}}\right)} .
$$

We first consider the positive solution for $p_{x}$. The expansion to first order in $\varepsilon$ is

$$
p_{x}=\sqrt{x_{0}^{2}+p_{x_{0}}^{2}-x^{2}-p_{y}^{2}-\frac{y^{2}}{q^{2}}}+\frac{\varepsilon\left(q^{2} x^{2}+y^{2}\right)^{2}}{8 q^{4} \sqrt{x_{0}^{2}+p_{x_{0}}^{2}-x^{2}-p_{y}^{2}-\frac{y^{2}}{q^{2}}}}+\mathcal{O}\left(\varepsilon^{2}\right),
$$

The equations of motion (16) on the energy level $H=h$ are given by $(22)$

$$
\begin{aligned}
\dot{x} & =\sqrt{x_{0}^{2}+p_{x_{0}}^{2}-x^{2}-p_{y}^{2}-\frac{y^{2}}{q^{2}}}+\frac{\varepsilon\left(q^{2} x^{2}+y^{2}\right)^{2}}{8 q^{4} \sqrt{x_{0}^{2}+p_{x_{0}}^{2}-x^{2}-p_{y}^{2}-\frac{y^{2}}{q^{2}}}}+\mathcal{O}\left(\varepsilon^{2}\right), \\
\dot{y} & =p_{y} \\
\dot{p}_{y} & =-\frac{y}{q^{2}}+\frac{\varepsilon y}{2 q^{4}}\left(q^{2} x^{2}+y^{2}\right)+\mathcal{O}\left(\varepsilon^{2}\right) .
\end{aligned}
$$


Now the differential system (22) has the form of (8), where

$$
F_{0}\left(x, y, p_{y}\right)=\left(\sqrt{x_{0}^{2}+p_{x_{0}}^{2}-x^{2}-p_{y}^{2}-\frac{y^{2}}{q^{2}}}, p_{y},-\frac{y}{q^{2}}\right),
$$

and

$$
F_{1}\left(x, y, p_{y}\right)=\left(\frac{\left(q^{2} x^{2}+y^{2}\right)^{2}}{8 q^{4} \sqrt{x_{0}^{2}+p_{x_{0}}^{2}-x^{2}-p_{y}^{2}-\frac{y^{2}}{q^{2}}}}, 0, \frac{y\left(q^{2} x^{2}+y^{2}\right)}{2 q^{4}}\right)
$$

with the unperturbed solution $\left(x_{0} \cos t+p_{x_{0}} \sin t, 0,0\right)$. Note that the order $\left\{x, y, p_{y}\right\}$ in the system (22) is important to accomplish the hypothesis (ii) of the Averaging Theorem. Here $k=1, n=3, \alpha=x_{0}, \mathbf{z}_{\alpha}=\left(x_{0}, 0,0\right)$ is the initial condition of the unperturbed periodic orbit, and for each $\mathbf{z}_{x_{0}}$ the solution $\mathbf{x}\left(t, \mathbf{z}_{x_{0}}\right)=\left(x_{0} \cos t+p_{x_{0}} \sin t, 0,0\right)$ is $2 \pi$-periodic.

Now we compute the linearization of the unperturbed system along the periodic solution $D_{\mathbf{x}} F_{0}\left(t, \mathbf{x}\left(t, \mathbf{z}_{x_{0}}\right)\right)$

$$
\left(\begin{array}{ccc}
-\frac{x_{0} \cos t+p_{x_{0}} \sin t}{p_{x_{0}} \cos t-x_{0} \sin t} & 0 & 0 \\
0 & 0 & 1 \\
0 & -\frac{1}{q^{2}} & 0
\end{array}\right)
$$

and the fundamental matrix is obtained solving (10)

$$
M_{\mathbf{z}_{x_{0}}}(t)=\left(\begin{array}{ccc}
\cos t-\frac{x_{0} \sin t}{p_{x_{0}}} & 0 & 0 \\
0 & \cos (t / q) & q \cos (t / q) \\
0 & -\frac{\sin (t / q)}{q} & \cos (t / q)
\end{array}\right),
$$

which satisfies $M_{\mathbf{z}_{x_{0}}}(0)=I$, and the inverse is given by

$$
M_{\mathbf{z}_{x_{0}}}^{-1}(t)=\left(\begin{array}{ccc}
\frac{p_{x_{0}}}{p_{x_{0}} \cos t-x_{0} \sin t} & 0 & 0 \\
0 & \cos (t / q) & -q \sin (t / q) \\
0 & \frac{\sin (t / q)}{q} & \cos (t / q)
\end{array}\right) .
$$


In order to apply the Averaging Theorem, we verify the condition det $\Delta_{x_{0}} \neq$ 0 , thus we compute

$$
M_{\mathbf{z}_{x_{0}}}^{-1}(0)-M_{\mathbf{z}_{x_{0}}}^{-1}(2 \pi)=\left(\begin{array}{ccc}
0 & 0 & 0 \\
0 & 2 \sin ^{2}(\pi / q) & q \sin (2 \pi / q) \\
0 & -\sin (2 \pi / q) / q & 2 \sin ^{2}(\pi / q)
\end{array}\right) .
$$

In the upper right corner, the $1 \times 2$ matrix is zero, and for each $\mathbf{z}_{x_{0}}$ in the lower right corner the matrix $\Delta_{x_{0}}$ has determinant non zero, $\operatorname{det} \Delta_{x_{0}}=$ $4 \sin ^{2}(\pi / q)$.

The function $F_{1}$ along the periodic orbit is given by

$$
F_{1}\left(t, \mathbf{x}\left(t, \mathbf{z}_{x_{0}}\right)\right)=\left(\frac{\left(x_{0} \cos t+p_{x_{0}} \sin t\right)^{4}}{8\left(p_{x_{0}} \cos t-x_{0} \sin t\right)}, 0,0\right),
$$

and we must apply to it the inverse of the fundamental matrix

$$
M_{\mathbf{z}_{x_{0}}}^{-1}(t) F_{1}\left(t, \mathbf{x}\left(t, \mathbf{z}_{x_{0}}\right)\right)=\left(\frac{p_{x_{0}}\left(x_{0} \cos t+p_{x_{0}} \sin t\right)^{4}}{8\left(p_{x_{0}} \cos t-x_{0} \sin t\right)^{2}}, 0,0\right) .
$$

The function $\mathcal{F}\left(x_{0}\right)$ defined in (11) is the projection $\xi$ in the first component of the integral of (28) in one period

$$
\mathcal{F}\left(x_{0}\right)=\int_{0}^{2 \pi} \frac{p_{x_{0}}\left(x_{0} \cos t+p_{x_{0}} \sin t\right)^{4}}{8\left(p_{x_{0}} \cos t-x_{0} \sin t\right)^{2}} d t=-\frac{3}{8} \pi p_{x_{0}}\left(x_{0}^{2}+p_{x_{0}}^{2}\right)
$$

where $p_{x_{0}}= \pm \sqrt{2 h-x_{0}^{2}}$ at the energy level (20), thus

$$
\mathcal{F}\left(x_{0}\right)=-\frac{3}{4} \pi h \sqrt{2 h-x_{0}^{2}} .
$$

Now we look for the zeros of $\mathcal{F}\left(x_{0}\right)=0: x_{0}= \pm \sqrt{2 h}$, which implies $p_{x_{0}}=0$. Every simple zero of $\mathcal{F}\left(x_{0}\right)$ provides a periodic orbit for the perturbed differential system in the energy level $H=h>0$. Finally, the negative solution of $(21)$ provides the same solutions than the positive one because $p_{x_{0}}=0$. Note that both initial conditions $( \pm \sqrt{2 h}, 0,0,0)$ provides the same periodic orbits. Theorem 1 is proved.

\section{Proof of Theorem 2}

This proof proceeds analogously as the previous one. Now the unperturbed periodic orbit lies on the $\left(0, y, 0, p_{y}\right)$ plane

$$
\begin{aligned}
x(t) & =0 \\
p_{x}(t) & =0 \\
y(t) & =y_{0} \cos (t / q)+p_{y_{0}} q \sin (t / q), \\
p_{y}(t) & =\left(p_{y_{0}} q \cos (t / q)-y_{0} \sin (t / q)\right) / q,
\end{aligned}
$$


with the first integral (17) when $\varepsilon=0$ taking the value

$$
h=\frac{1}{2}\left(p_{y_{0}}^{2}+\frac{y_{0}^{2}}{q^{2}}\right) .
$$

We solve for $p_{y}$ from (17) at the energy level $H=h$ with $h$ given by $(32)$

$$
p_{y}= \pm \sqrt{\frac{y_{0}^{2}}{q^{2}}+p_{y_{0}}^{2}-x^{2}-p_{x}^{2}-\frac{y^{2}}{q^{2}}+\varepsilon\left(\frac{x^{4}}{4}+\frac{x^{2} y^{2}}{2 q^{2}}+\frac{y^{4}}{4 q^{4}}\right)} .
$$

We take $p_{x_{0}} \geq 0$.

The expansion up to first order in $\varepsilon$ is

$$
p_{y}=\sqrt{\frac{y_{0}^{2}}{q^{2}}+p_{y_{0}}^{2}-x^{2}-p_{x}^{2}-\frac{y^{2}}{q^{2}}}+\frac{\varepsilon\left(q^{2} x^{2}+y^{2}\right)^{2}}{8 q^{4} \sqrt{\frac{y_{0}^{2}}{q^{2}}+p_{y_{0}}^{2}-x^{2}-p_{x}^{2}-\frac{y^{2}}{q^{2}}}}+\mathcal{O}\left(\varepsilon^{2}\right),
$$

The equations of motion (16) on the energy level $H=h$ are (34)

$$
\begin{aligned}
\dot{x} & =p_{x}, \\
\dot{y} & =\sqrt{\frac{y_{0}^{2}}{q^{2}}+p_{y_{0}}^{2}-x^{2}-p_{x}^{2}-\frac{y^{2}}{q^{2}}}+\frac{\varepsilon\left(q^{2} x^{2}+y^{2}\right)^{2}}{8 q^{4} \sqrt{\frac{y_{0}^{2}}{q^{2}}+p_{y_{0}}^{2}-x^{2}-p_{x}^{2}-\frac{y^{2}}{q^{2}}}}+\mathcal{O}\left(\varepsilon^{2}\right), \\
\dot{p}_{x} & =-x+\frac{\varepsilon x}{2}\left(\frac{y^{2}}{q^{2}}+x^{2}\right)+\mathcal{O}\left(\varepsilon^{2}\right) .
\end{aligned}
$$

We must write the system in the order $\left\{y, x, p_{x}\right\}$ in the system (34) to accomplish the hypothesis (ii) in the Averaging Theorem, with $\alpha=y_{0}$, $\mathbf{z}_{y_{0}}=\left(y_{0}, 0,0\right)$ and the solution $\mathbf{x}\left(t, \mathbf{z}_{y_{0}}\right)=\left(y_{0} \cos (t / q)+p_{y_{0}} \sin (t / q), 0,0\right)$ being $2 \pi q$-periodic.

The linearization of the unperturbed system along the periodic solution is

$$
D_{\mathbf{x}} F_{0}\left(t, \mathbf{x}\left(t, \mathbf{z}_{y_{0}}\right)\right)=\left(\begin{array}{ccc}
-\frac{y_{0} \cos (t / q)+p_{y_{0}} q \sin (t / q)}{q\left(p_{y_{0}} q \cos (t / q)-y_{0} \sin (t / q)\right)} & 0 & 0 \\
0 & 0 & 1 \\
0 & -1 & 0
\end{array}\right),
$$


and the fundamental matrix is obtained solving (10)

$$
M_{\mathbf{z}_{y_{0}}}(t)=\left(\begin{array}{ccc}
\cos (t / q)-\frac{y_{0} \sin (t / q)}{p_{y_{0}} q} & 0 & 0 \\
0 & \cos t & \sin t \\
0 & -\sin t & \cos t
\end{array}\right)
$$

which satisfys $M_{\mathbf{z}_{y_{0}}}(0)=I$, with the inverse

$$
M_{\mathbf{z}_{y_{0}}}^{-1}(t)=\left(\begin{array}{ccc}
\frac{p_{y_{0}} q}{p_{y_{0}} q \cos (t / q)-y_{0} \sin (t / q)} & 0 & 0 \\
0 & \cos t & -\sin t \\
0 & \sin t & \cos t
\end{array}\right) .
$$

In order to apply the Averaging Theorem we compute

$$
M_{\mathbf{z}_{y_{0}}}^{-1}(0)-M_{\mathbf{z}_{y_{0}}}^{-1}(2 \pi q)=\left(\begin{array}{ccc}
0 & 0 & 0 \\
0 & 2 \sin ^{2}(\pi q) & \sin (2 \pi q) \\
0 & -\sin (2 \pi q) & 2 \sin ^{2}(\pi q)
\end{array}\right) .
$$

In the upper right corner the $1 \times 2$ matrix is zero, and in the lower right corner the $2 \times 2$ matrix $\Delta_{y_{0}}$ has

$$
\operatorname{det} \Delta_{x_{0}}=4 \sin ^{2}(\pi q) \neq 0
$$

and we can apply the averaging method.

The function $F_{1}$ along the periodic orbit is given by

$$
F_{1}\left(t, \mathbf{x}\left(t, \mathbf{z}_{y_{0}}\right)\right)=\left(\frac{\left(y_{0} \cos (t / q)+p_{y_{0}} q \sin (t / q)\right)^{4}}{8 q^{3}\left(p_{y_{0}} q \cos (t / q)-y_{0} \sin (t / q)\right)}, 0,0\right)
$$

and then

(39) $M_{\mathbf{z}_{y_{0}}}^{-1}(t) F_{1}\left(t, \mathbf{x}\left(t, \mathbf{z}_{y_{0}}\right)\right)=\left(\frac{p_{y_{0}}\left(y_{0} \cos (t / q)+p_{y_{0}} q \sin (t / q)\right)^{4}}{8 q^{2}\left(p_{y_{0}} q \cos (t / q)-y_{0} \sin (t / q)\right)^{2}}, 0,0\right)$.

The function $\mathcal{F}\left(y_{0}\right)$ defined in (11) as the first component of the integral in a period $2 \pi q$ of (39) is given by

$$
\mathcal{F}\left(y_{0}\right)=-\frac{3}{4} \pi h q \sqrt{2 h-\frac{y_{0}^{2}}{q^{2}}}
$$

where we have used that $p_{y_{0}}=\sqrt{2 h-y_{0}^{2} / q^{2}}$ at the fixed energy level (32). Every simple zero $y_{0}= \pm \sqrt{2 h q}$ of $\mathcal{F}\left(y_{0}\right)$ provides a periodic orbit for the perturbed differential system in the energy level $H=h>0$. But since the initial conditions $(0,0, \pm \sqrt{2 h q}, 0)$ provide the same periodic orbit, Theorem 2 follows. It is easy to realize that the two signs in (33) provide the same initial conditions $(0,0, \pm \sqrt{2 h q}, 0)$ of the periodic orbit. 
As we mentioned before, the case $q$ rational was also studied in cartesian coordinates but we did not obtain information because we could not solve the variational equations. We also tried to work with a generalization of polar coordinates, but we could not get information in this case from the averaging method.

\section{Proof of Theorem 3}

This section follows essentially from section 4 of the reference [8]. We summarize some facts on the Liouville-Arnold integrability of the Hamiltonian systems, and on the theory of the periodic orbits of the differential equations. See $[1,3]$ and the subsection 7.1.2 of [3]. We present these results for Hamiltonian systems of two degrees of freedom, but these results work for an arbitrary number of degrees of freedom.

We recall that a Hamiltonian system with Hamiltonian $H$ of two degrees of freedom is integrable in the sense of Liouville-Arnold if it has a first integral $C$ independent with $H$ (i.e. the gradient vectors of $H$ and $C$ are independent in all the points of the phase space except perhaps in a set of zero Lebesgue measure), and in involution with $H$ (i.e. the parenthesis of Poisson of $H$ and $C$ is zero). For Hamiltonian systems with two degrees of freedom the involution condition is redundant, because the fact that $C$ is a first integral of the Hamiltonian system, implies that the mentioned Poisson parenthesis is always zero. A flow defined on a subspace of the phase space is complete if its solutions are defined for all time.

Now we state the Liouville-Arnold Theorem $[1,3]$ restricted to Hamiltonian systems of two degrees of freedom.

Liouville-Arnold Theorem. Suppose that a Hamiltonian system with two degrees of freedom defined on the phase space $M$ has its Hamiltonian $H$ and the function $C$ as two independent first integrals in involution. If $I_{h c}=\{p \in M: H(p)=h$ and $C(p)=c\} \neq \emptyset$ and $(h, c)$ is a regular value of the differentiable map $(H, C)$, then the following statements hold.

(a) $I_{h c}$ is a two dimensional submanifold of $M$ invariant under the flow of the Hamiltonian system.

(b) If the flow on a connected component $I_{h c}^{*}$ of $I_{h c}$ is complete, then $I_{h c}^{*}$ is diffeomorphic either to the torus $\mathbb{S}^{1} \times \mathbb{S}^{1}$, or to the cylinder $\mathbb{S}^{1} \times \mathbb{R}$, or to the plane $\mathbb{R}^{2}$. If $I_{h c}^{*}$ is compact, then the flow on it is always complete and $I_{h c}^{*} \approx \mathbb{S}^{1} \times \mathbb{S}^{1}$.

(c) Under the hypothesis (b) the flow on $I_{h c}^{*}$ is conjugated to a linear flow on $\mathbb{S}^{1} \times \mathbb{S}^{1}$, on $\mathbb{S}^{1} \times \mathbb{R}$, or on $\mathbb{R}^{2}$. 
We consider the autonomous differential system

$$
\dot{x}=f(x),
$$

where $f: U \rightarrow \mathbb{R}^{n}$ is $C^{2}, U$ is an open subset of $\mathbb{R}^{n}$ and the dot denotes the derivative with respect to the time $t$. We write its general solution as $\phi\left(t, x_{0}\right)$ with $\phi\left(0, x_{0}\right)=x_{0} \in U$ and $t$ belonging to its maximal interval of definition.

We say that $\phi\left(t, x_{0}\right)$ is $T$-periodic with $T>0$ if and only if $\phi\left(T, x_{0}\right)=$ $x_{0}$ and $\phi\left(t, x_{0}\right) \neq x_{0}$ for $t \in(0, T)$. The periodic orbit associated to the periodic solution $\phi\left(t, x_{0}\right)$ is $\gamma=\left\{\phi\left(t, x_{0}\right), t \in[0, T]\right\}$. The variational equation associated to the $T$-periodic solution $\phi\left(t, x_{0}\right)$ is

$$
\dot{M}=\left(\left.\frac{\partial f(x)}{\partial x}\right|_{x=\phi\left(t, x_{0}\right)}\right) M,
$$

where $M$ is an $n \times n$ matrix. The monodromy matrix associated to the $T$ periodic solution $\phi\left(t, x_{0}\right)$ is the solution $M\left(T, x_{0}\right)$ of $(42)$ satisfying that $M\left(0, x_{0}\right)$ is the identity matrix. The eigenvalues $\lambda$ of the monodromy matrix associated to the periodic solution $\phi\left(t, x_{0}\right)$ are called the multipliers of the periodic orbit. For an autonomous differential system, one of the multipliers is always 1 , the corresponding eigenvector is tangent to the periodic orbit, and another is 1 due to the existence of the first integral given by the Hamiltonian.

The following theorem of Poincaré [14] gives us a method for proving that the non Liouville-Arnold integrability, based on the existence of the two periodic orbits of Theorems 1 and 2, must be for any second first integral of class $\mathcal{C}^{1}$.

Poincaré Theorem. If a Hamiltonian system with two degrees of freedom and Hamiltonian $H$ is Liouville-Arnold integrable, and $C$ is a second first integral such that the differentials of $H$ and $C$ are linearly independent at each point of a periodic orbit of the system, then all the multipliers of this periodic orbit are equal to 1.

The main problem to use this theorem is to show analytically the existence of periodic orbits having multipliers different from 1.

Proof of Theorem 3. We assume that we are under the assumptions of Theorems 1 and 2, i.e. $q$ is irrational and the energy level is positive. Then there exist the two periodic orbits given by Theorems 1 and 2 . Moreover their associated Jacobians (12) are different from 1, in fact, they go to infinity. Since the Jacobians are the product of the multipliers of these periodic orbits, all the multipliers cannot be equal to 1. Hence, 
if $q$ is irrational and $H=h>0$, by the Poincaré Theorem, either the cored and logarithmic Hamiltonian systems cannot be Liouville-Arnold integrable with any second first integral $C$, or the system is LiouvilleArnold integrable and the differentials of $H$ and $C$ are linearly dependent at some point for every periodic orbit of Theorems 1 and 2. Therefore the theorem is proved.

\section{Conclusions}

We introduced a small parameter by means of a non-canonical scale transformation, which allowed to us to study Hamiltonian systems near Liouville-Arnold integrable ones, in our case near the bidimentional harmonic oscillator. We have used averaging theory to show the existence of two families of periodic orbits of the cored and logarithmic Hamiltonian systems, and we gave analytically their initial conditions up to first order in $\varepsilon>0$ in their fixed positive energy levels. Then we have used these periodic orbits and the Poincaré Theorem to study that the nonintegrability in the sense of Liouville-Arnold of those two Hamiltonian systems.

\section{ACKNOWLEDGMENTS}

We thank the comments and suggestions of the referee which help us to improve the presentation of this article.

The second author is partially supported by a MICINN/FEDER grant number MTM 2008-03437, by an AGAUR grant number 2009SGR 410 and by ICREA Academia.

\section{REFERENCES}

[1] Abraham R. and Marsden J.E., Foundations of Mechanics, Benjamin, Reading, Masachusets, 1978.

[2] Arnol'd V.I., Forgotten and neglected theories of Poincaré, Russian Math. Surveys 61 No. 1 (2006), 1?-18.

[3] Arnol'd V.I., Kozlov V. and Neishtadt A., Dynamical Systems III. Mathematical Aspects of Classical and Celestial Mechanics, third edition, Encyclopaedia of Mathematical Science, Springer, Berlin, 2006.

[4] Belmonte C., Boccaletti D. and Pucacco G., On the Structure of the logarithmic potential, The Astrophysical Journal 669 (2007), 202-217.

[5] Buică A., Francoise J. and Llibre J., Periodic solutions of nonlinear periodic differential systems with a small parameter, Communications on pure and applied analysis 6 (2007), 103-111.

[6] Contopoulos G. and Seimenis J. Application of the Prendergast method to a logarithmic potential, Astronomy and Astrophysics, 227 (1990), 49-53. 
[7] Kozlov V.V., Integrability and non-integrability in Hamiltonian mechanics, Russian Math. Surveys 38 No. 1 (1983), 1-76.

[8] Jiménez-Lara L. and Llibre, J., Periodic orbits and non-integrability of generalized classical Yang-Mills Hamiltonian systems, Journal of Mathematical Physics 52 (2011), 032901 pp. 9.

[9] Llibre J. and Jiménez-Lara L., Periodic orbits and non-integrability of HenonHeiles systems, Journal of Physics A, Math. and Theor. 44 (2011), 205103 pp. 14.

[10] Papaphilippou Y. and Laskar J., Frequency map analysis and global dynamics in a two degrees of freedom galactic potential, Astronomy and Astrophysics, 307 (1996), 427-449.

[11] Pucacco G., Boccaletti D. and Belmonte C., Quantitative predictions with detuned normal forms, Celestial Mechanics and Dynamical Astronomy 102 (2008), 163-176.

[12] Pucacco G., Boccaletti D. and Belmonte C., Periodic orbits in the logarithmic potential, Astronomy and Astrophysics, 489 (2008), 1055-1063.

[13] Malkin, I.G., Some problems of the theory of nonlinear oscillations, (Russian) Gosudarstv. Izdat. Tehn.- Teor. Lit., Moscow, 1956.

[14] Poincaré H., Les méthodes nouvelles de la mécanique céleste, Vol. I, GauthierVillars, Paris 1899.

[15] Roseau M., Vibrations non linéaires et théorie de la stabilité, (French) Springer Tracts in Natural Philosophy, 8. Springer, Berlin Heidelberg New York, 1966.

[16] Sanders, J. A., Verhultst, F. and Murdock, J. Averaging Methods in Nonlinear Dynamical Systems, Applied Mathematical Sci. 59, Springer Verlag, New York, rev. ed., 2007.

[17] Scuflaire R., Stability of axial orbits in analytical galactic potentials, Celestial Mechanics and Dynamical Astronomy 61 (1995), 261-285.

[18] Verhulst, F. Nonlinear Differential Equations and Dynamical Systems, Springer Verlag, 1996.

${ }^{1}$ Departamento de Física, Universidad Autónoma Metropolitana-Iztapalapa, P.O. Box 55-534, MÉxico, D.F., 09340 MÉxico

E-mail address: lidia@xanum.uam.mx

${ }^{2}$ Departament de Matemàtiques, Universitat Autònoma de Barcelona, 08193 Bellaterra, Barcelona, Catalonia, Spain

E-mail address: jllibre@mat.uab.cat 